\title{
Chronic Tenosynovitis of the Upper Extremities Caused by Mycobacterium kansasii: A Clinical Case and Systematic Review of Literature
}

\author{
Maria T. Huayllani ${ }^{1}$ Andrea Sisti ${ }^{1}$ Daniel Boczar ${ }^{1}$ David J. Restrepo ${ }^{1}$ Alexander S. Parker ${ }^{2}$ \\ Rachel Sarabia-Estrada ${ }^{3}$ Brian D. Rinker ${ }^{1} \quad$ Antonio Jorge Forte ${ }^{1, \odot}$
}

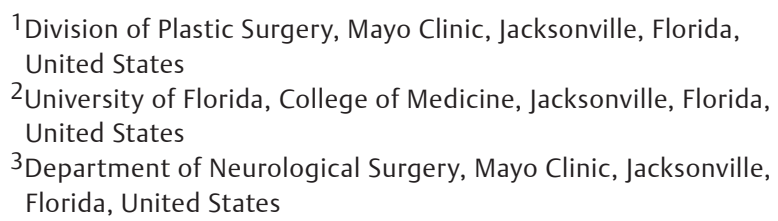

Address for correspondence Antonio Jorge Forte, MD, PhD, 4500 San Pablo Road, Jacksonville, FL 32224, United States (e-mail: ajvforte@yahoo.com.br).

Indian J Plast Surg 2020;53:25-35

\begin{abstract}
Keywords

- chronic tenosynovitis

- Mycobacterium kansasii

- characteristics

- complications

- hand

- upper extremity
\end{abstract}

Background Chronic tenosynovitis of the upper extremities caused by Mycobacterium kansasii (M. kansasii) is uncommon, but symptoms may overlap with other more common diseases. Late diagnosis and treatment can lead to disfiguration of structures and rupture of tendons, resulting in worse cosmetic outcomes after reconstruction.

Methods We present a clinical case and literature review of $M$. kansasii in patients with chronic tenosynovitis of upper extremities. PubMed was queried for cases of upper extremities tenosynovitis caused by M. kansasii. The keywords "M. kansasii," "tenosynovitis" and synonyms were used for search in different combinations. Manuscripts, with no specific data or another condition, where the infection was not located in the upper extremities, were reviews, or not in English, were excluded from the study.

Results We described 23 reported cases of tenosynovitis of the upper extremity caused by $M$. kansasii. An immunosuppressed state was present in eight (34.8\%) cases, and 12 (52.2\%) patients received immunosuppressive treatment. A long-time period between the first appearance of symptoms and the definitive diagnosis was identified (median: 7 months, interquartile range: 9 ). The most frequent symptoms were local swelling (65.2\%), pain (56.5\%), mass effect (26\%), and stiffness (13\%). Tendon rupture was found in three $(13 \%)$ patients as a complication of the disease. Moreover, seven (30.4\%) patients underwent previous surgeries to try to relieve the symptoms before definitive diagnosis was achieved.

Conclusion M. kansasii is an important differential causal pathogen for tenosynovitis of the upper extremities. Although rare, raising awareness about this infectious disease is imperative to avoid inadequate management and hazardous aesthetic sequelae.

\section{Introduction}

Extrapulmonary infection of nontuberculosis Mycobacterium (NTM) is rare. ${ }^{1}$ It could affect lymph nodes, skin, genitourinary, and musculoskeletal systems, causing chronic infections in humans. ${ }^{2,3}$ Chronic tenosynovitis is a rarely described
DOI https://doi.org/ 10.1055/s-0040-1709377 ISSN 0970-0358. presentation of NTM infection, especially in developed countries like the United States. ${ }^{3}$

Mycobacterium kansasii (M. kansasii) is the second most common NTM, principally affecting the hand, and the most frequent NTM infection of the joints and synovia, ${ }^{1}$ even though the rate of frequency is five times lower 
than Mycobacterium marinum. ${ }^{4}$ It is considered an acid-fast and slow growing mycobacterium that takes a long time to appear in cultures ${ }^{2,4}$; accordingly, the diagnosis is difficult in early stages. Furthermore, immunosuppressed patients are more prone to develop an infection by M. kansasii.,

It is important to be aware that NTM could cause tenosynovitis of the hand, especially in the context of immunosuppression, to treat it promptly and preserve the structure and function of the hands. To date, the specific disease characteristics, complications, and treatment of chronic tenosynovitis caused by M. kansasii are not well-known. For that reason, we aim to present a case report and to perform a systematic review that describes the characteristics, complications, and approach related to chronic tenosynovitis caused by M. kansasii.

\section{Clinical Case}

A 64-year-old man with a 5-year history of treatment-resistant rheumatoid arthritis (RA) presented with a 10-month history of pain and swelling of his left wrist. He had been hospitalized 1 year prior for development of drug rash with eosinophilia and systemic symptoms to sulfasalazine, requiring treatment with a short course of prednisone. Two months later, he developed a warm mass on the dorsal surface of his left wrist with restricted range of motion on extension (-Fig. 1). Rheumatology prescribed hydroxychloroquine 200-mg daily, followed by a short course of corticosteroids, as the symptoms were attributed to RA flare. Magnetic resonance imaging (MRI) showed a circumferential heterogeneous collection of fluid in the extensor tendons compartment at the level of the wrist (-Fig. 2). The diagnosis was severe active tenosynovitis of the extensors attributed to RA, given the patient's history. For the next 6 months, the mass had a fast growth and then leveled off, despite treatment. Rheumatology prescribed different biologic therapies, such as adalimumab, abatacept, and tocilizumab, without improvement of the tenosynovitis.

The patient was referred to our department to exclude other diagnoses that might have caused the tenosynovitis. He underwent a left dorsal hand extensor tenosynovectomy involving the extensor pollicis longus, extensor carpi radialis longus, extensor carpi radialis brevis, extensor digitorum communis, extensor indicis proprius, and extensor digiti minimi. A dorsal incision was performed exposing a very large mass on the dorsum of the hand composed of inflamed synovium and fluid, which were sent to pathology for analysis (-Fig. 3). Tocilizumab therapy was stopped 2 weeks before surgery, but he continued using prednisone
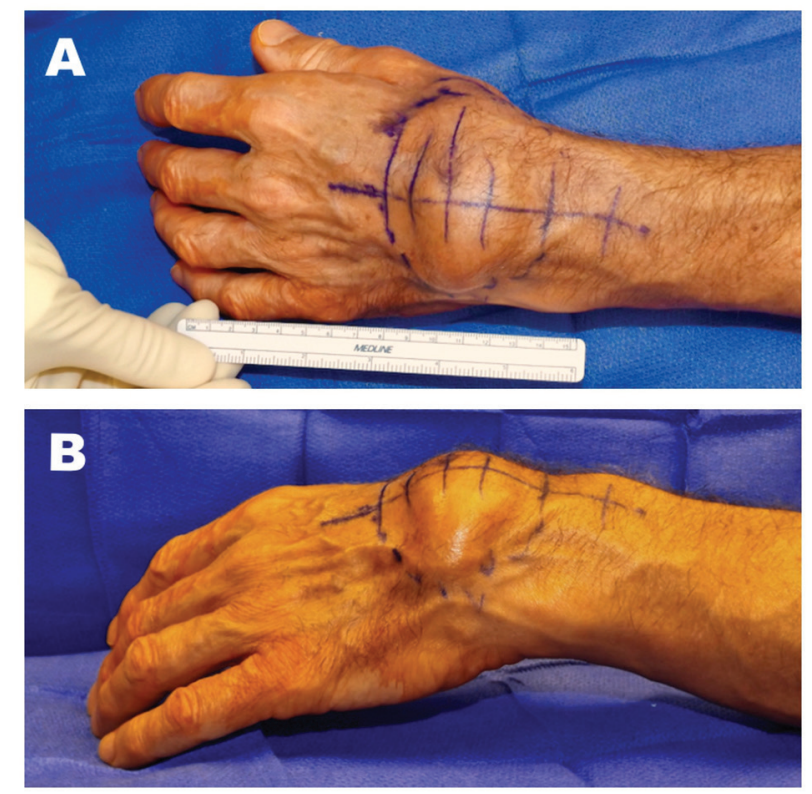

Fig. 1 Left hand appearance. (A) Dorsal view of the mass on left wrist. (B) Side view of the mass on left wrist.
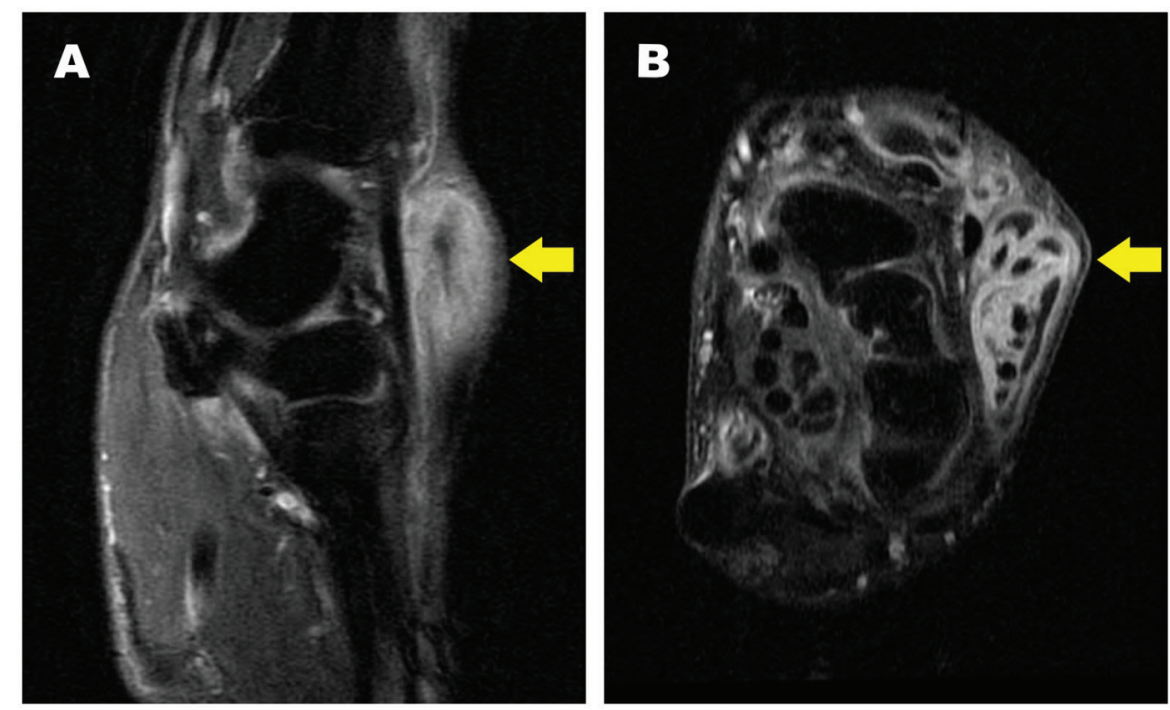

Fig. 2 Gadolinium-enhanced and fat-suppressed magnetic resonance imaging of the left wrist, demonstrating considerable signs of inflammation and fluid surrounding the extensor tendons. (A) Sagittal view of the left wrist. (B) Axial view of the left wrist. Yellow arrows point to the specific site of inflammation. 

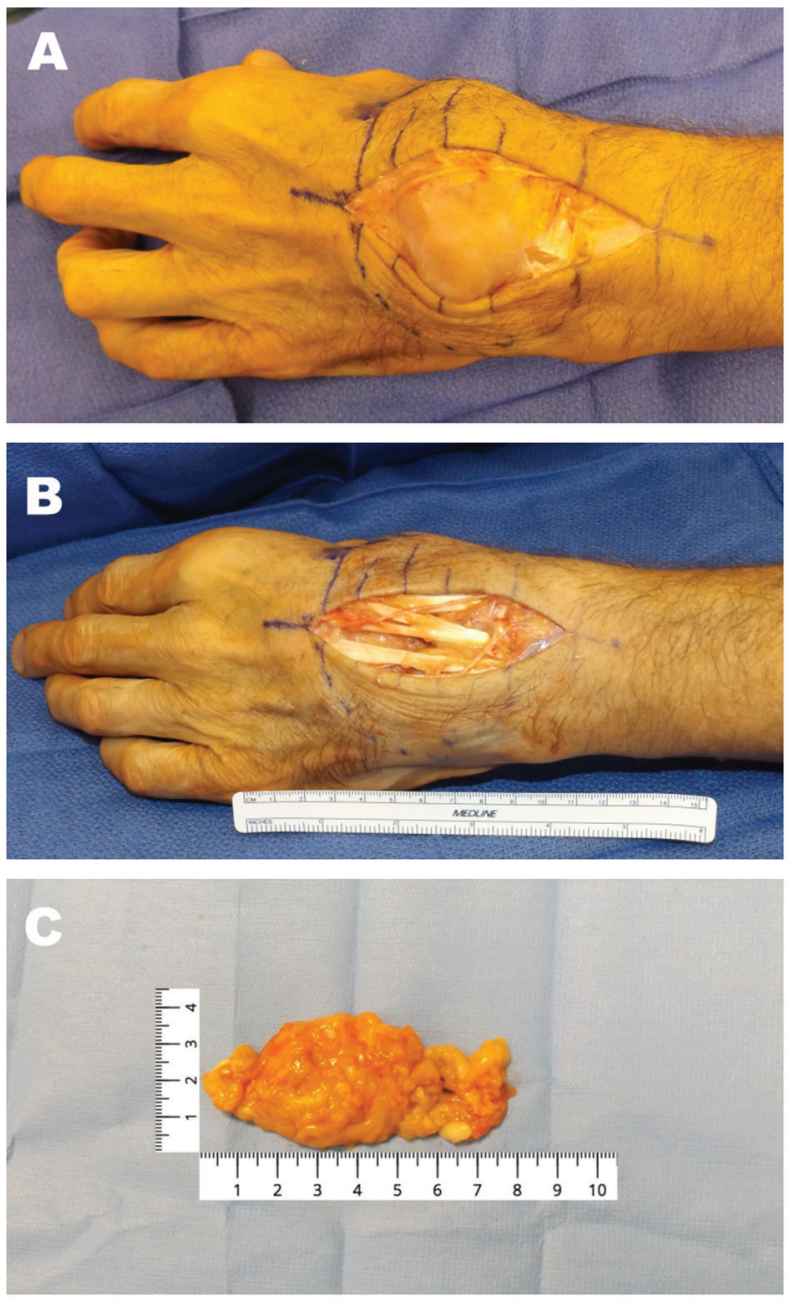

Fig. 3 Intraoperative left hand appearance. (A) Exposed left wrist synovia. (B) Extensor tendons of the left wrist exposed. (C) Surgical specimen.

5-mg daily. Laboratory analysis showed the following results: hemoglobin, $14.4 \mathrm{~g} / \mathrm{dL}$; hematocrit, $40.8 \%$; white blood cell

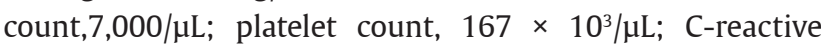
protein, $<3.0 \mathrm{mg} / \mathrm{L}$; c-antineutrophil cytoplasmic antibody, negative; $p$-antineutrophil cytoplasmic antibody, negative; antinuclear antibodies, $0.4 \mathrm{U}$; Smith antibody immunoglobulin $\mathrm{G}$, $<0.2 \mathrm{U}$; rheumatoid factor, $<15 \mathrm{IU} / \mathrm{mL}$; and cyclic citrullinate peptide antibody, $<15.6 \mathrm{IU} / \mathrm{mL}$.

Pathology results of the synovial biopsy demonstrated a moderate chronic inflammation and many epithelioid granulomas with minimal necrosis. Acid-fast bacilli (AFB) and Gomori methenamine silver stains were negative for AFB and fungal microorganisms, respectively, and bacterial and fungal cultures were negative. AFB culture was positive for M. kansasii, with three colonies reported 1 month after surgery, and was negative by probe for Mycobacterium tuberculosis and Mycobacterium avium complex.

After the procedure, his left hand surgical site healed, with some minimal residual swelling, and specialized therapy was performed to recover hand function ( - Fig. 4). Initial empirical therapy was azithromycin 500-mg daily, ciprofloxacin
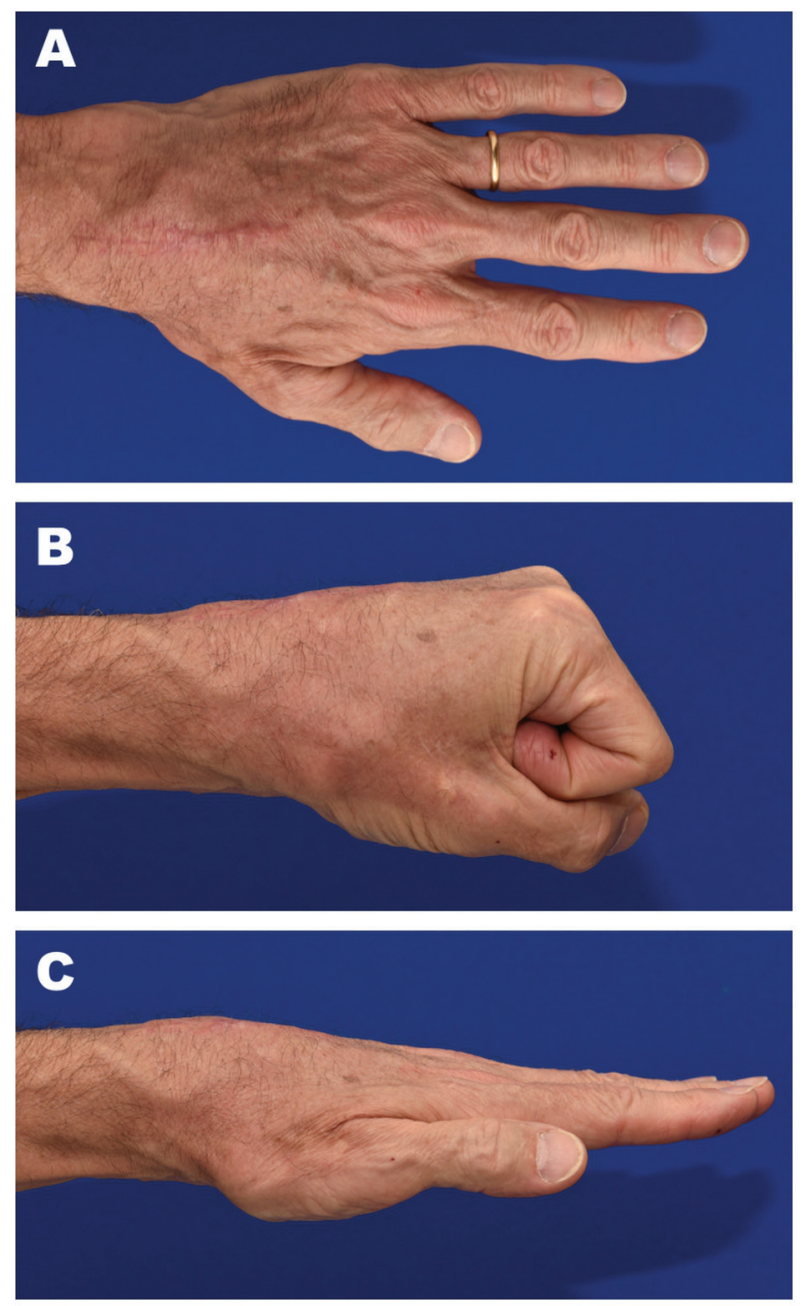

Fig. 4 Postoperative images, 5 weeks after surgery. (A) Dorsal view of the left hand showing a $85-\mathrm{mm}$ scar. (B) Left hand making a fist. (C) Side view of the left hand.

500-mg twice daily, and doxycycline 100-mg twice daily. When the final results of AFB culture were reported, the therapy was changed to ethambutol 1,200-mg daily, rifampin 600-mg daily, and azithromycin 500-mg daily to be given for 3 months until the following visit. The patient's left hand was progressing well after a few days; however, he decided to continue treatment and follow-up in his hometown.

\section{Systematic Review}

\section{Methods}

A PICo model (population, interest, context) for a qualitative study was constructed to determine the characteristics, complications, and treatment of chronic tenosynovitis caused by M. kansasii in the upper extremities. The PubMed database was queried for cases of upper extremities chronic tenosynovitis caused by M. kansasii. A search strategy was generated using the following MESH terms: "tenosynovitis" OR "tenosynovitis/arthritis" OR "tenosynovitis/case" OR "tenosynovitis/ case reports" OR "tenosynovitis/in" OR "tenosynovitis/localized" OR "tenosynovitis case reports" OR "tenosynovitis cases" 
OR "tenosynovitis complications" OR "tenosynovitis conditions" AND "M. kansasii" OR "kansasii" OR "Mycobacterium," with last access on October 25, 2019. Articles were selected for their accuracy in anatomical localization in the upper extremities and with confirmed diagnosis. Identified articles were uploaded into EndNote and screened manually by the first author (M.T.H.) and selected according to the inclusion and exclusion criteria. If there was any doubt in the selection of one article, the second author (A.S.) reviewed the manuscript, and both reviewers came to an agreement for the final decision. Articles written in English were included. Exclusion criteria were manuscripts where there were no specific data regarding $M$. kansasii infection, were reviews, or were written in a language other than English. Relevant data were extracted and presented as follows: author, year, sex, age, time period between symptoms and diagnosis, past medical history, immunocompromised status, symptoms, site, initial treatment, diagnose confirmation, complications, definitive treatment, antibiotic time, and outcome. As there was substantial heterogeneity in the reported data, a quantitative analysis could not be conducted and was not the goal of this review. Bias assessment was not performed since the publications included were all case reports.

\section{Results}

Our initial search query resulted in a total of 26 articles. Excluding manuscripts with cases of tenosynovitis with no specific data for M. kansasii, other conditions different than tenosynovitis, and other locations different than the upper extremities by screening on titles and abstracts yielded 20 articles. Including only English language articles, and excluding reviews that did not describe any case, we identified 17 articles, from which 23 cases of chronic tenosynovitis of the upper extremities caused by M. kansasii were reported (-Fig. 5). All the articles were published between 1978 and 2018. We described characteristics and complications of the 23 reported cases in total ( - Table $\mathbf{1}$ ). Most of the patients were female (52.2\%), with a female: male ratio of 1.09 and a mean age of 54.74 (standard deviation: 12.18) years. An antecedent of trauma was present in three (13\%) patients ${ }^{5-7}$ and in one
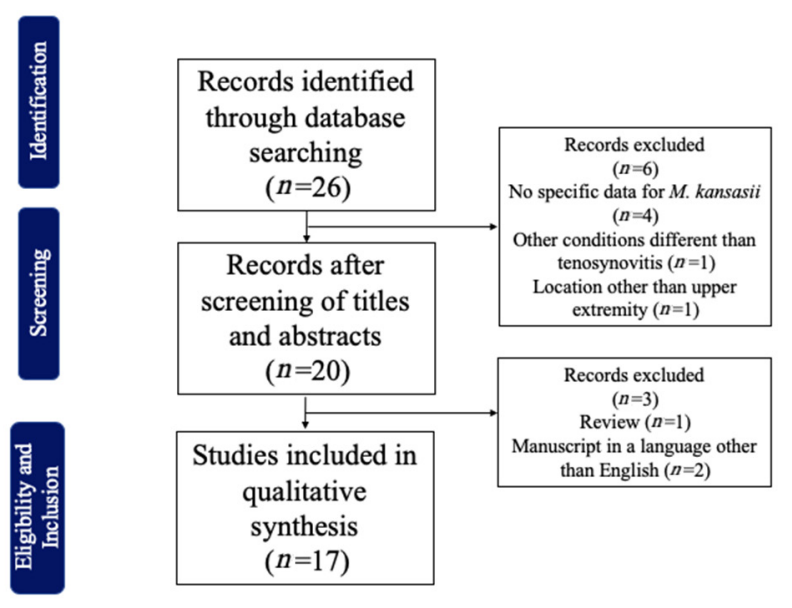

Fig. 5 Flow chart of search strategy and articles identification. patient, ${ }^{8}$ a history of chemical spill in the affected hand was described. An immunosuppression state was usual but not the most frequent immune state in patients with this infection, presenting in only eight (34.8\%) cases..$^{19-15}$ The median of the time period between the first appearing of symptoms and the definitive diagnosis was 7 months (interquartile range: 9 , range: $2-48$ ). The most frequent symptoms were local swelling (65.2\%), ${ }^{1,6,8,10-14,16-19}$ pain (56.5\%), ${ }^{1,6,7,9,10,12,15,18-20}$ mass effect (26\%),5,12,13,15 and stiffness (13\%)., ${ }^{7,9}$ Twelve (52.2\%) patients received an initial immunosuppressive therapy. . $^{131,6-8,13,18-20}$ Two $(8.7 \%)$ of these patients received this treatment due to similar presentation to their previous RA disease ${ }^{1,13}$ and one $(4.3 \%)$ due to previous episodes of joints pain. ${ }^{19}$ Other immunosuppressive medical conditions included polymyositis, ${ }^{9}$ diabetes mellitus, ${ }^{10,12}$ scleroderma, ${ }^{11}$ kidney transplantation, ${ }^{1,15}$ and rheumatic polymyalgia. ${ }^{14}$ Furthermore, four (17.4\%) patients presented a carpal tunnel syndrome as initial disease presentation. ${ }^{10,16,19,20}$ Tendon rupture was found in three (13\%) patients as a complication of the disease. ${ }^{1,18,19}$ All the diagnoses were confirmed with a microbiological culture of the tissue. Regarding treatment, seven (30.4\%) patients underwent previous surgeries before definitive diagnosis to try to relieve the symptoms. ${ }^{6,7,10,12,13,19}$ Antibiotic therapy was given in all cases, with the most common regimen being rifampin, ethambutol, isoniazid, and pyridoxine for long time periods (mean: 13.27 months, range: $1-24$ ) to avoid recurrence of the disease. Resistance to isoniazid was found in two patients, ${ }^{10,19}$ two patients stopped treatment due to adverse effects to ethambutol, ${ }^{14,16}$ one to rifampin, ${ }^{6}$ and one to clarithromycin. ${ }^{14}$ Definitive treatment such as tenosynovectomy, surgical debridement, synovial biopsy, and antibiotic therapy alone were performed in ten $(43.5 \%),{ }^{7,8,10,13,15-17,19,20}$ nine (39.1\%), ${ }^{1,6,9,12,18}$ two $(8.7 \%),{ }^{19}$ and two $(12.5 \%)^{11,14}$ cases, respectively. The rate of recurrence of symptoms in patients with tenosynovectomy was $50 \%$ whereas in patients who underwent surgical debridement was $22 \%$. No recurrence was described in patients treated only with antibiotics. All cases reported at the end a positive response to therapy.

\section{Discussion}

Our review of literature found the most important disease characteristics of chronic tenosynovitis of the upper extremity caused by M. kansasii. M. kansasii was found in high rates in the southern United States. ${ }^{4}$ The bacteria has been found in soil, natural water supplies, dust and animal milk, ${ }^{13,17}$ although in contrast to other common NTM, the major reservoir of $M$. kansasii appears to be tap water and people likely acquire it by aerosol or trauma. ${ }^{2}$ In our review, an antecedent that suggests mode of transmission was not described for almost all cases. This creates an impression that transmission by aerosol could be predominant over trauma as only two cases reported the antecedent of trauma and one of chemical spill in the hand where the tenosynovitis appeared. Regarding other antecedents, more than half of the patients were immunocompetent, which means that even when immunosuppression state predisposes to prompt dissemination of the nontuberculous mycobacteria due to the decrease 


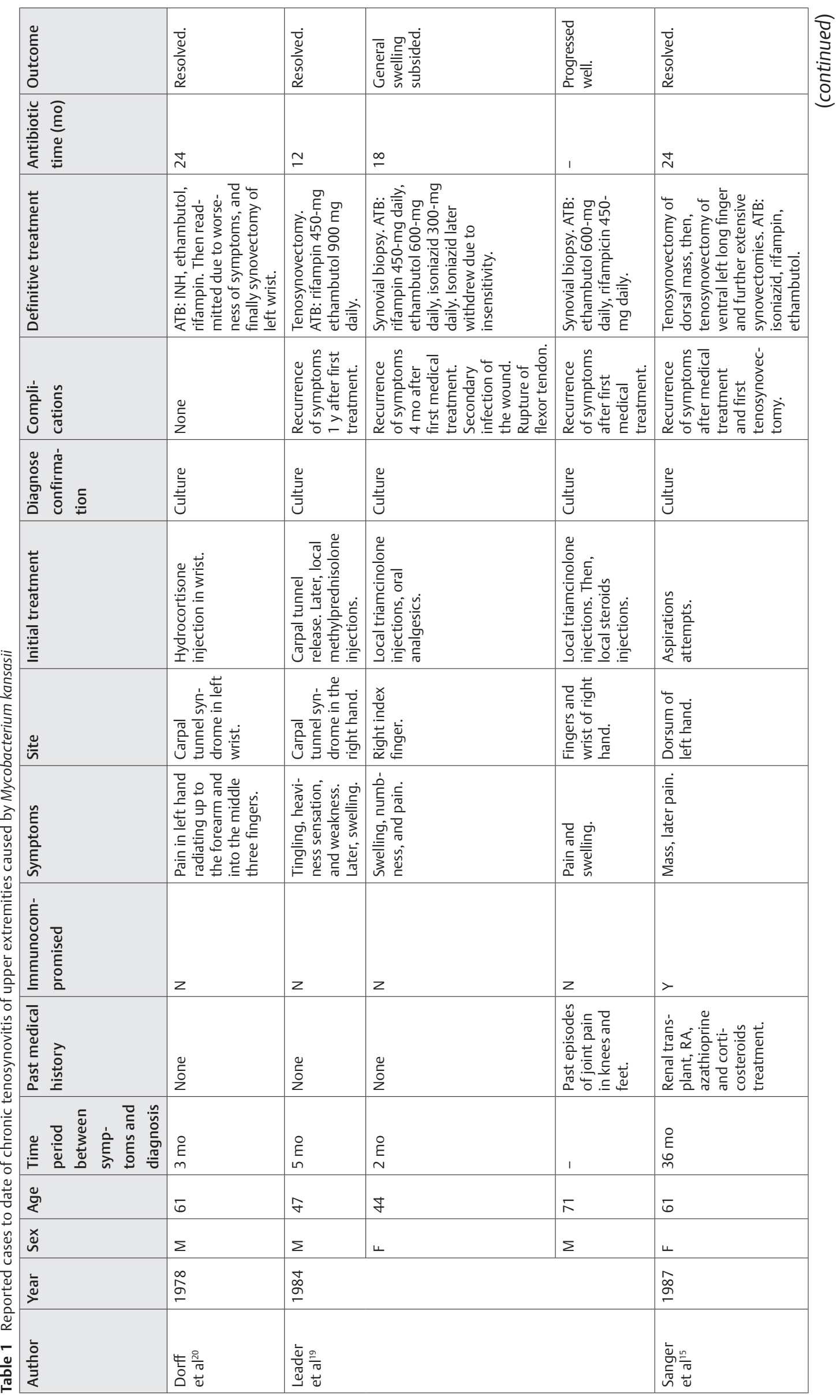




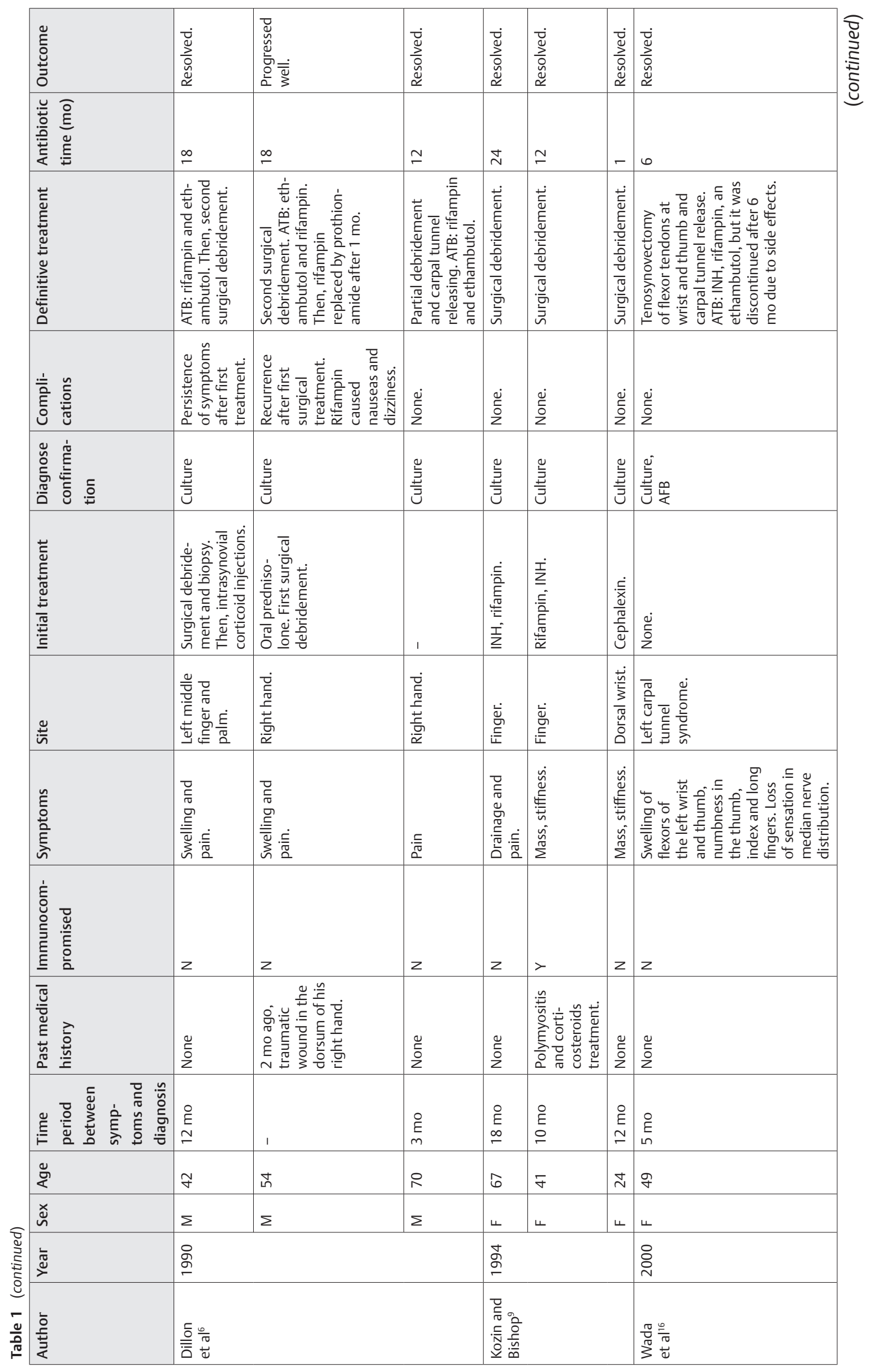




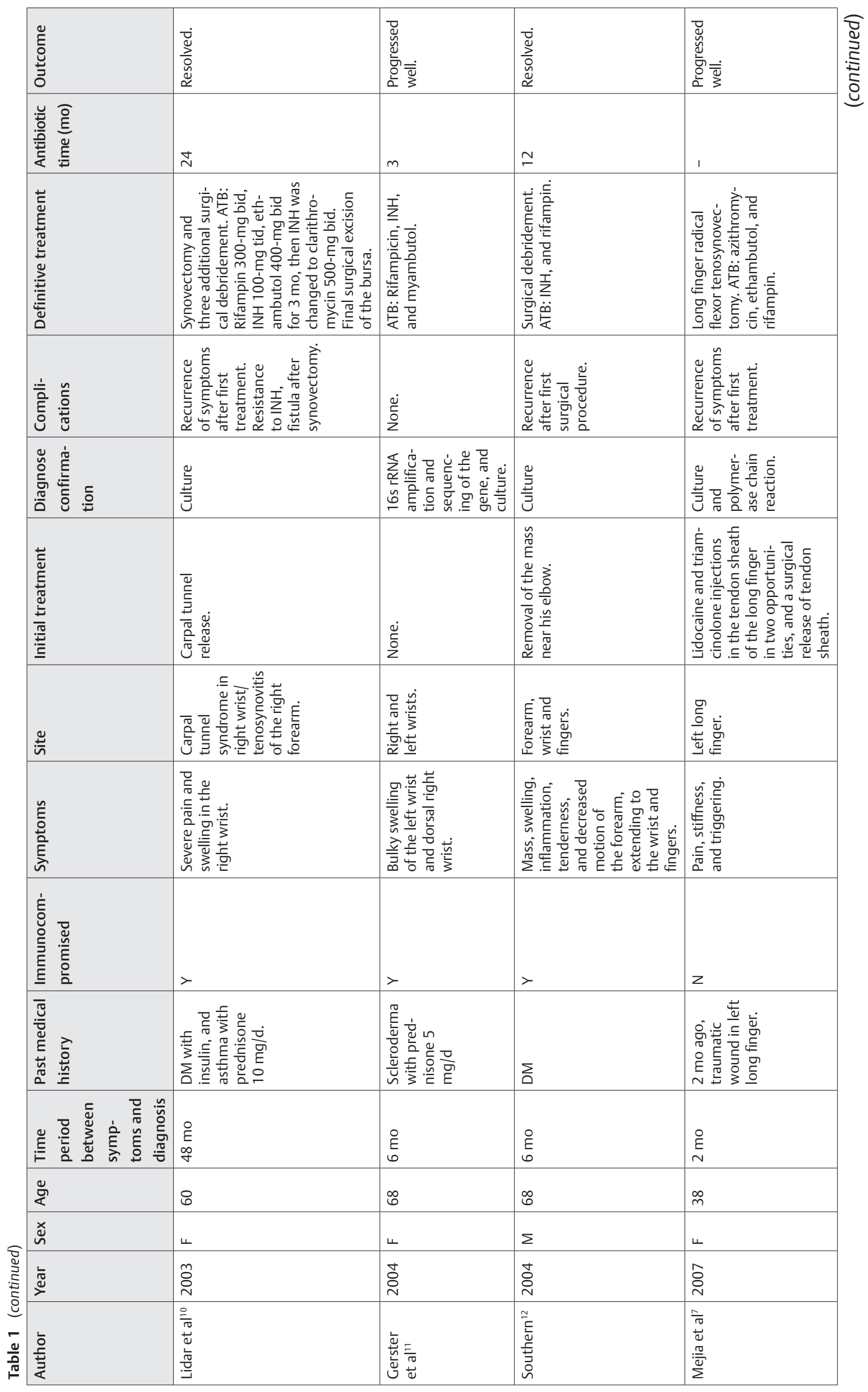




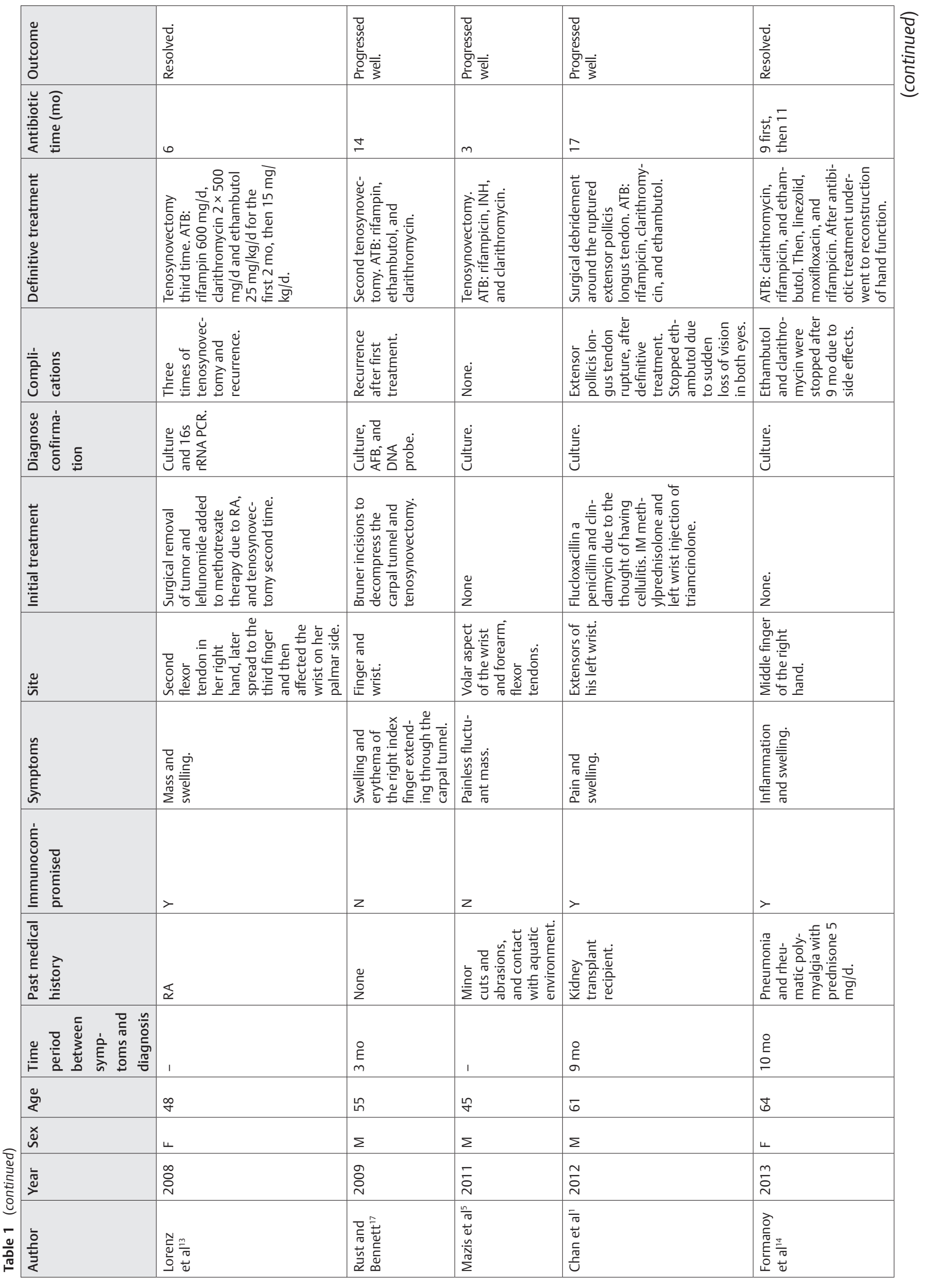




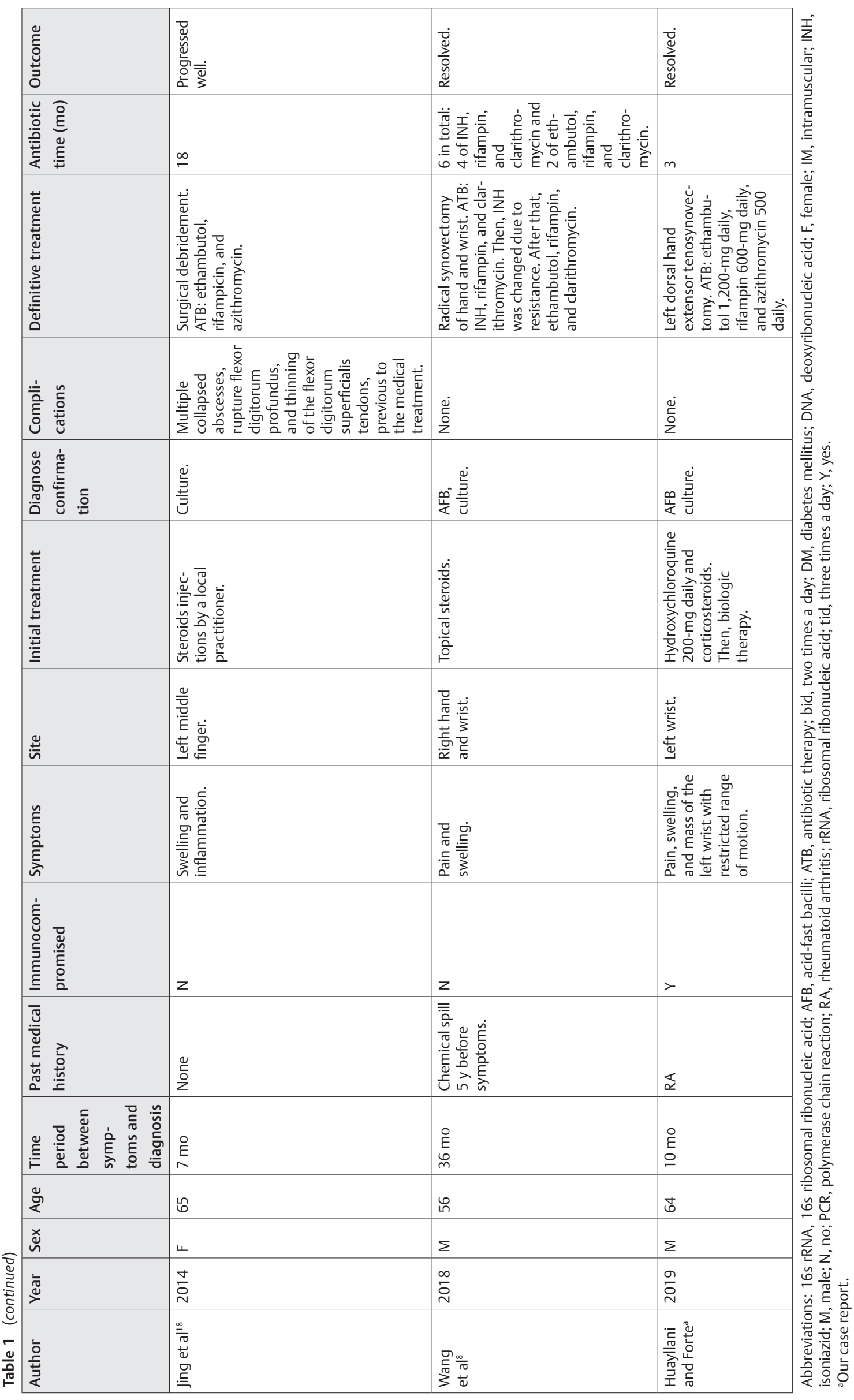


of inflammatory mediators action, ${ }^{17,21}$ immune state may not be a strong factor that determines bacteria acquisition.

The median of the period time between appearing of symptoms and definitive diagnosis was 7 months. This finding suggests the insidious presentation of the disease and the delayed diagnosis probably caused by the nonspecific symptoms such as swelling, pain, mass effect, and stiffness ${ }^{1,5,7,9,13,18-20,22}$ that can be confused to be an expression of other conditions such as carpal tunnel syndrome, ${ }^{10,16,19,20}$ previous history of joints pain, ${ }^{19}$ or RA. .13

First immunosuppressive therapy was given in almost half of the patients probably because of attribution of symptoms to tenosynovitis caused by a history of RA or joints pain ${ }^{1,13,19}$ or mechanical causes, ${ }^{6-8,18,20}$ or as a therapy for a previous immunologic condition. ${ }^{9,11,14,15}$ This therapy could worsen the infection and could have been predisposed to tendon rupture in three cases. ${ }^{1,18,19}$

Regarding diagnosis, all the cases confirmed their diagnosis through culture. We found that almost one-third of the patients underwent previous surgeries before definitive diagnosis, some of them may be the result of false negatives after performing other diagnostic techniques or due to an incorrect sample taking of synovial tissue and fluid for culture. Moreover, NTM characterizes long periods of culture which can delay the definitive diagnosis. ${ }^{2,4}$ Stains for acid-fast bacteria alone usually do not definitively diagnose pathology. Culture of the bacteria has a high sensitivity, and it should be considered the preferred diagnostic method. ${ }^{1,13}$ Polymerase chain reaction and DNA hybridization tests are recommended to shorten time to diagnosis, but negative results do not exclude the infection. ${ }^{13}$ Between the imaging studies, we recommend when possible the use of MRI as it helped acquire a better idea of the disease extension such in the presented case.

Interestingly, the medical and surgical treatment strategy differed between studies. There is no consensus on therapy for M. kansasii chronic tenosynovitis. All patients who underwent any type of surgical treatment were also treated with antibiotics. We found a rate of recurrence of symptoms of $50 \%$ in patients who had tenosynovectomy and $22 \%$ in patients who had surgical debridement as a definitive treatment. A promising approach is to perform a tenosynovectomy in combination with surgical debridement in these cases. However, future studies with higher sample sizes should be performed to provide stronger evidence that support a potential benefit. No recurrence was found after synovial biopsy and in patients who only were treated with antibiotics. Previously, drug therapy alone or tenosynovectomy alone was attempted, but the frequency of recurrence was high. Between the cases treated only with antibiotics, one of them ${ }^{9}$ was followed until 3 months progressing well but a longer period of follow-up is needed to evaluate better outcomes, while the other case ${ }^{12}$ had to undergo reconstruction of hand after a long-time treatment with antibiotics which suggests a worse hand function outcome. Therefore, after reviewing the presence of complications and number of surgical procedures, we strongly recommend a combination of surgical tenosynovectomy and antibiotic chemotherapy. The Infectious Diseases Society of America (IDSA) and American Thoracic Society (ATS) guidelines recommend a combination of rifampin, ethambutol, isoniazid, and pyridoxine for 18 months for pulmonary involvement, until cultures are negative for 12 months. ${ }^{2}$ Although this recommendation was not established in a tenosynovitis scenario, most of the previous cases used some of these antibiotics to treat the bacteria in a chronic tenosynovitis presentation. ${ }^{1,13}$ Due to the lack of guidelines specifying the treatment for NTM chronic tenosynovitis, therapy is usually adapted to the patient and based on individual expert opinion to avoid recurrence. As a result, many cases have shown no recurrence with the use of the antibiotics recommended by the IDSA-ATS guidelines with long (18 months) and short (3-6 months) periods of treatment. ${ }^{23}$

The therapy applied in the reported case included azithromycin, rifampin, and ethambutol and it was recommended for 3 months with the possibility to extend the therapy depending on the progression of the disease, until 18 months. We recommend this antibiotic regimen in combination with thorough follow-up by a hand therapist. This antibiotic regimen has been reported in tenosynovitis of the finger caused by M. kansasii, with good results. ${ }^{18}$ It is also important to provide close follow-up, when feasible, to address any recurrence.

\section{Strengths and Limitations}

Limitations of our study included a possible publication bias which made difficult defining a specific successful treatment for this condition. In addition, inherent limitations of a review methodology can involve search and selection biases that should be also considered. However, we believe this review is valuable as it is the first study that reports all the cases, treatments, and complications of chronic tenosynovitis in the upper extremities caused by M. kansasii.

\section{Conclusion}

kansasii is an important differential causal pathogen to consider in tenosynovitis of the upper extremities. Our review identified that the mode of transmission of the bacteria was not clear in most of the cases, and it was usual to infect immunocompromise patients; however, it was more common to appear in immunocompetent patients. Moreover, immunosuppressive states may predispose development of tendon rupture, considered the worst complication. The diagnosis of the disease was usually late due to its insidious progression and unspecific symptoms; however, when symptoms appear this potential infectious agent should be ruled out as a potential cause of tenosynovitis. Early diagnosis of chronic tenosynovitis caused by $M$. kansasii can be challenging based on the clinical presentation; however, prompt detection and treatment will avoid morbidity and possible structural complications. 


\section{Funding Source}

This study was supported in part by the Mayo Clinic Center for Individualized Medicine and the Plastic Surgery Foundation.

\section{Conflict of Interest}

The reports by A.S.P., A.S., A.J.F., B.D.R., D.B., D.J.R., M.T.H., R.S.-E. and this study were supported in part by the Mayo Clinic Robert D. and Patricia E. Kern Center for the Science of Health Care Delivery, by the Mayo Clinic Center of Individualized Medicine, and by the Plastic Surgery Foundation.

\section{Acknowledgment}

None.

\section{References}

1 Chan A, Findlay A, Abeygunasekara S. A case of wrist tenosynovitis caused by Mycobacterium kansasii in a renal transplant recipient. Transpl Infect Dis 2012;14(5):E44-E49

2 Johnston JC, Chiang L, Elwood K. Mycobacterium kansasii. Microbiol Spectr 2017;5(1):5

3 Balagué N, Uçkay I, Vostrel P, Hinrikson H, Van Aaken I, Beaulieu JY. Non-tuberculous mycobacterial infections of the hand. Chir Main 2015;34(1):18-23

4 Zenone T, Boibieux A, Tigaud S, et al. Non-tuberculous mycobacterial tenosynovitis: a review. Scand J Infect Dis 1999;31(3):221-228

5 Mazis GA, Sakellariou VI, Kontos F, Zerva L, Spyridonos SG. Recurrent fluctuant mass of the wrist and forearm associated with chronic tenosynovitis by Mycobacterium kansasii. Orthopedics 2011;34(5):400

6 Dillon J, Millson C, Morris I. Mycobacterium kansasii infection in the wrist and hand. Br J Rheumatol 1990;29(2):150-153

7 Mejia H, Ryzewicz M, Scott F. Trigger finger due to tenosynovitis from Mycobacterium kansasii infection in an immunocompetent patient. Orthopedics 2007;30(12):1055-1056

8 Wang MS, Berry M, Lehto-Hoffman A, Vi L, Ramessar N. Chronic tenosynovitis due to Mycobacteria kansasiiin an immunocompetent host. Case Rep Infect Dis 2018;2018:3297531
9 Kozin SH, Bishop AT. Atypical Mycobacterium infections of the upper extremity. J Hand Surg Am 1994;19(3):480-487

10 Lidar M, Elkayam O, Goodwin D, Yaron M, Caspi D. Protracted Mycobacterium kansasii carpal tunnel syndrome and tenosynovitis. Isr Med Assoc J 2003;5(6):453-454

11 Gerster JC, Duvoisin B, Dudler J, Berner IC. Tenosynovitis of the hands caused by Mycobacterium kansasii in a patient with scleroderma. J Rheumatol 2004;31(12):2523-2525

12 Southern PM Jr. Tenosynovitis caused by Mycobacterium kansasii associated with a dog bite. Am J Med Sci 2004;327(5):258-261

13 Lorenz HM, Dalpke AH, Deboben A, et al. Mycobacterium kansasii tenosynovitis in a rheumatoid arthritis patient with long-term therapeutic immunosuppression. Arthritis Rheum 2008;59(6):900-903

14 Formanoy E, Lam HY, Arends JE. Tenosynovitis of the right handMycobacterium kansasii. Neth J Med 2013;71(10):526-530

15 Sanger JR, Stampfl DA, Franson TR. Recurrent granulomatous synovitis due to Mycobacterium kansasii in a renal transplant recipient. J Hand Surg Am 1987;12(3):436-441

16 Wada A, Nomura S, Ihara F. Mycobacterium kansasiiflexor tenosynovitis presenting as carpal tunnel syndrome. J Hand Surg [Br] 2000;25(3):308-310

17 Rust PA, Bennett T. An uncommon cause for a common complaint. Hand Surg 2009;14(2-3):131-134

18 Jing SS, Teare L, Iwuagwu F. Mycobacterium kansasii flexor tenosynovitis of the finger. Hand Surg 2014;19(2):249-251

19 Leader M, Revell P, Clarke G. Synovial infection with Mycobacterium kansasii. Ann Rheum Dis 1984;43(1):80-82

20 Dorff GJ, Frerichs L, Zabransky RJ, Jacobs P, Spankus JD. Musculoskeletal infections due to Mycobacterium kansasii. Clin Orthop Relat Res 1978;(136):244-246

21 Campbell IA, Ormerod LP. "Opportunist" mycobacterial infections. Thorax 2000;55(8):722

22 Saraya T, Fukuoka K, Maruno H, et al. Tenosynovitis with rice body formation due to mycobacterium intracellular infection after initiation of infliximab therapy. Am J Case Rep 2018;19:656-662

23 Yano K, Yoshida T, Minoda Y, et al. Clinical outcome of the chronic flexor tenosynovitis in the hand caused by non-tuberculous mycobacterium treated by extensive tenosynovectomy and drugs. J Plast Surg Hand Surg 2013;47(6):434-437 